\title{
Programa de detección del alelo APOE-E4 en adultos mayores mexicanos con deterioro cognitivo
}

\author{
Alma Delia Genis-Mendoza, ${ }^{1}$ José Jaime Martínez-Magaña, ${ }^{1}$ Carolina Bojórquez, ${ }^{1}$ \\ José Alberto Téllez-Martínez, ${ }^{1}$ Janett Jiménez-Genchi, ${ }^{2}$ Andrés Roche, ${ }^{2}$ Alexis Bojorge, ${ }^{2}$ \\ Mariana Chávez, ${ }^{2}$ Carlos Castañeda, ${ }^{2}$ Rafael Guzmán, ${ }^{3}$ Leonor Zapata, ${ }^{3}$ Dione Aguilar-Méndez, ${ }^{3}$ \\ Nuria Lanzagorta, ${ }^{4}$ Ileana Rebolledo, ${ }^{4}$ Susana Castro-Chavira, ${ }^{5}$ Thalía Fernández, ${ }^{5}$ Lorena Orozco, ${ }^{6}$ \\ Humberto Nicolini ${ }^{1}$ y Angélica Graciela Martínez-Hernández ${ }^{6}$ \\ ${ }^{1}$ Secretaría de Salud, Instituto Nacional de Medicina Genómica, Laboratorio de Genómica de Enfermedades Psiquiátricas y Neurodegenerativas, \\ Ciudad de México, México; ${ }^{2}$ Secretaría de Salud, Hospital "Fray Bernardino Álvarez", Servicios de Atención Psiquiátrica, Ciudad de México, México; \\ ${ }^{3}$ Secretaría de Salud, Hospital General de México, Clínica de Geriatría, Ciudad de México, México; ${ }^{4}$ Grupo Médico Carracci, Ciudad de México, \\ México; ${ }^{5}$ Universidad Nacional Autónoma de México, Instituto de Neurología, Querétaro, México; ${ }^{6}$ Secretaría de Salud, Instituto Nacional de \\ Medicina Genómica, Laboratorio de Inmunogenómica y Enfermedades Metabólicas, Ciudad de México, México
}

\begin{abstract}
Resumen
Introducción: En México, la prevalencia de los trastornos neurocognitivos (TNC) han aumentado a la par del incremento en la esperanza de vida. El alelo E4 del gen que codifica la apolipoproteína $E$ (APOE) es el principal factor de riesgo genético para deterioro neurocognitivo. Objetivo: Reproducir la asociación en población mexicana entre APOE-E4 y el deterioro neurocognitivo, así como implementar un programa de detección de riesgo genético con el alelo APOE-E4. Método: Se estructuró un programa de detección de riesgo basado en APO-EA en diferentes centros de reclutamiento en la zona centro de la República Mexicana, con tres etapas: reclutamiento y selección de los candidatos para la detección del alelo de riesgo, análisis del riesgo genético y entrega del resultado. Resultados: El análisis de asociación genética para replicar la asociación con trastornos neurocognitivos mediante modelos logísticos multivariados mostró que el alelo E4 de APOE incrementó aproximadamente $6 \%$ el riesgo en población mexicana $(R M=5.83, p=0.0025)$. Se entregaron 367 resultados de riesgo genético. Conclusiones: El presente programa es el primero en México implementado para dar a conocer un factor de riesgo genético para trastornos neurocognitivos en varios centros del país.
\end{abstract}

PALABRAS CLAVE: Trastornos neurocognitivos. Marcadores genéticos. Deterioro cognitivo.

\begin{abstract}
Introduction: In Mexico, the prevalence of neurocognitive disorders (NCDs) has increased in parallel with the increase in life expectancy. The E4 allele of the gene that encodes apolipoprotein $E(A P O E)$ is the main genetic risk factor for cognitive impairment. Objective: To replicate the association of APOE-E4 allele with neurocognitive impairment in a Mexican population, as well as to implement a genetic risk-detection program with the APOE-E4 allele. Method: $A$ program was structured for the detection of APOE-E4 allele risk in different recruiting centers from the central zone of the Mexican Republic, with three stages: recruitment and selection of candidates for the detection of the risk-allele, genetic risk analysis and delivery of results. Results: In the genetic-association study to replicate the association with neurocognitive disorders by means of multivariate logistic models, the APOE-E4 allele increased the risk for cognitive impairment in the Mexican populations by approximately
\end{abstract}

Fecha de recepción: 12-10-2017

Fecha de aceptación: 23-04-2018

DOI: 10.24875/GMM.18003784
Gac Med Mex. 2018;154:555-560

Disponible en PubMed

www.gacetamedicademexico.com 
$6 \%$ (OR: 5.83, $p=0.0025)$. In addition, 367 genetic risk results were delivered. Conclusions: The present program is the first one to be implemented in Mexico with the purpose to inform on a genetic risk factor for neurocognitive disorders in several centers of the country.

KEY WORDS: Neurocognitive disorders. Genetic markers. Cognitive impairment.

\section{Introducción}

La Organización Mundial de la Salud estima que aproximadamente 35.6 millones de personas en el mundo presentan diagnóstico de algún tipo de trastorno neurocognitivo (TNC) y se prevé que este número se duplique cada 20 años. En México, el Estudio Nacional sobre Salud y Envejecimiento en México (Enasem) reportó que $7 \%$ de la población mexicana cursaba con algún tipo de TNC menor (anteriormente denominado deterioro cognitivo leve) y $3 \%$ presentaba datos clínicos compatibles con TNC mayor (demencia). ${ }^{1-3}$

De acuerdo con el Manual Diagnóstico y Estadístico de los Trastornos Mentales en su quinta edición (DSM-5), los TNC pueden ser divididos en mayores y menores, conforme el nivel de preservación o afectación de la capacidad funcional; el deterioro cognitivo no deberá estar presente desde el nacimiento o tempranamente en la vida y deberá involucrar por lo menos dos o más dominios cognitivos (atención, funciones ejecutivas, aprendizaje y memoria, lenguaje, habilidades visuoperceptivas, cognición social).

A partir de estos criterios, dicho manual reconoce los siguientes TNC: asociado con la enfermedad de Alzheimer (EA), frontotemporal, con cuerpos de Lewy, vascular, asociado con traumatismo craneoencefálico, infección por el virus de la inmunodeficiencia humana, enfermedad de Huntington, enfermedad de Parkinson, enfermedad por priones, o inducido por sustancias, de etiología múltiples, así como no especificado (American Psychiatric Association 2013). De todos los anteriores, el TNC asociado con EA es el de mayor prevalencia.

El impacto económico que representan los TNC en el mundo asciende a cerca de 604 billones de dólares, casi la mitad del producto interno bruto de México. ${ }^{4}$ Por este motivo y el alarmante incremento en su prevalencia, los TNC principalmente por EA representan un problema de salud pública en el mundo y México.

Aun cuando la etiología de la EA no se ha establecido con claridad, se han identificado diversos factores que incrementan el riesgo de su aparición: inactividad física, tabaquismo, hipertensión arterial, obesidad, diabetes, depresión, bajo nivel educativo y algunas mutaciones a nivel genético. ${ }^{5}$ Investigaciones clínicas, principalmente en países con altos ingresos económicos como Estados Unidos y el Reino Unido, han sugerido que combatir estos factores de riesgo y diagnosticar de forma temprana a los individuos en alto riesgo tendrían un impacto positivo en la reducción de la prevalencia de padecer TNC tipo EA.6,7

El objetivo de generar un conjunto de herramientas que permitan agrupar a los individuos con alto riesgo de desarrollar EA ha sido el punto de convergencia de investigaciones recientes. En este sentido se han desarrollado diversas evaluaciones clínicas y cognitivas, así como marcadores en líquido cefalorraquídeo, a nivel de neuroimagen y genético. ${ }^{8}$ Sin embargo, como sucede en otras enfermedades, algunos han sido replanteados debido a su alto costo, además de que la mayoría es observable cuando la enfermedad se encuentra en etapas avanzadas. ${ }^{9}$

Los marcadores genéticos han presentado buenos valores de predicción para la enfermedad de Alzheimer, principalmente el alelo E4 de la apolipoproteína E (APOE). ${ }^{10}$ Se sabe que ser portador de una copia del alelo E4 de APOE incrementa tres veces el riesgo de EA; mientras que ser portador de dos copias del alelo puede acrecentarlo hasta 15 veces. ${ }^{11,12}$ La asociación entre el gen APOE-E4 y enfermedad de Alzheimer ha sido replicada tantas veces que la Food and Drug Administration en Estados Unidos aprobó su uso como marcador de riesgo genético para este TNC (https://www.fda.gov/newsevents/newsroom/pressannouncements/ucm551185.htm).

Sin embargo, en México y en numerosos países de ingresos económicos medios y bajos aún no se han establecido programas de tamizaje genético que permitan detectar a los individuos en alto riesgo de padecer EA o algún otro tipo de TNC. En 2015, Gutiérrez Robledo et al. planteaban que en México era urgente generar programas que permitieran identificar a los individuos con alto riesgo de demencia, para instaurar estrategias de intervención en esta población. ${ }^{13}$ Tomando como base esta premisa, presentamos un programa colaborativo entre distintas instituciones mexicanas para la 
detección de individuos en alto riesgo genético para TNC, al cual hemos denominado "Te APOyo".

\section{Método}

El programa de detección de APOE-E4 "Te APOyo" se estructuró en tres etapas.

\section{Reclutamiento y selección de los candidatos para la detección del alelo de riesgo}

Los pacientes fueron evaluados por médicos psiquiatras, psicólogos o geriatras con entrenamiento en TNC. El personal clínico decidía si el paciente era candidato para continuar con la evaluación del alelo de riesgo. Los criterios de inclusión fueron ser mayor de 60 años, contar con autorreporte de deterioro en el funcionamiento cognitivo basal, manifestado por queja subjetiva de pérdida de memoria 0 de algún otro dominio cognitivo como atención, funciones ejecutivas, lenguaje, habilidades visuoconstructivas, habilidades visuoperspectivas, cognición social o clinimetría compatible con deterioro cognitivo ( $<24$ puntos en la prueba Mini-Mental State Examination). ${ }^{14,15}$

Los sujetos fueron excluidos del estudio si presentaban condiciones que no permitían la adecuada evaluación clínica o clínimétrica: sintomatología psiquiátrica aguda (psicótica, ansiosa o afectiva), intoxicación o dependencia a sustancias o fármacos, discapacidad intelectual, daño cerebral agudo, entre otros. A todos los participantes se les pidió asentar por escrito su consentimiento para participar en la investigación, previa información y aclaración de dudas.

\section{Análisis del genotipo}

Previa valoración clínica, se consiguieron muestras biológicas de los sujetos como sangre periférica o raspado de células epiteliales de la parte interna de las mejillas. Las muestras biológicas solicitadas se emplearon para extraer el ADN, el cual se obtuvo mediante un método comercial modificado de salting-out, Gentra Puregene ${ }^{\circledR}$ (Qiagen), ajustando el protocolo de acuerdo con las especificaciones del proveedor para procesar las muestras.

La genotipificación de los polimorfismos del gen APOE (rs7412 y rs429358) se realizó mediante ensayos de reacción en cadena de la polimerasa en tiempo real, utilizando sondas Taqman $^{\circledR}$ (Applied
Biosystems, San Francisco, CA, EE. UU.). Para la genotipificación del polimorfismo rs429358 y rs7412 se utilizaron los ensayos C_3084793_20 y C_904973_10, respectivamente. Las condiciones de termociclado fueron las establecidas por el proveedor para cada ensayo. El termociclado y la discriminación alélica se realizaron en el equipo de tiempo real Quant Studio 6 Flex $^{\circledR}$ (Applied Biosystems, San Francisco, CA, EE. UU.

Una vez generados los resultados de riesgo genético, se procedió a reportarlos a cada paciente y a su cuidador primario, mediante un formato diseñado para la fácil comprensión de la información (Anexo 1).

\section{Entrega de resultados}

Aproximadamente después de un mes de la toma de muestra biológica se generó el resultado de riesgo genético. El médico tratante y un psicólogo entrenado en el manejo de pacientes con TNC entregaron personalmente los resultados a cada participante en presencia de un familiar o acompañante, mediante un formato diseñado para el protocolo y para cada uno de los centros de reclutamiento (Anexo 1).

\section{Análisis estadístico}

Con la finalidad de replicar la asociación de la $A P O E$ con el TNC se implementaron modelos logísticos multivariados, ajustados por edad y sexo. Los análisis se realizaron comparando las frecuencias alélicas de APOE-E3 con las de APOE-E4.

\section{Resultados}

El análisis de asociación genética replicó la asociación del alelo APOE-E4 con TNC en la población mexicana. A partir de población general, previamente se reclutaron 1307 individuos mestizos mexicanos, quienes fueron considerados como controles. ${ }^{16}$

Los participantes con genotipo homocigoto para APOE-E4 presentaron un factor de riesgo para TNC aproximadamente seis veces mayor (razón de momios, $R M=5.83$, [2.23-14.78] y $p=0.0025)$; en los heterocigotos para el alelo $\mathrm{E} 4$, el riesgo fue aproximadamente dos veces mayor $(\mathrm{RM}=1.78,[1.14-2.76]$ y $p=0.0104)$. En la Tabla 1 y Figura 1 se resumen las frecuencias alélicas y genotípicas de la población analizada. 


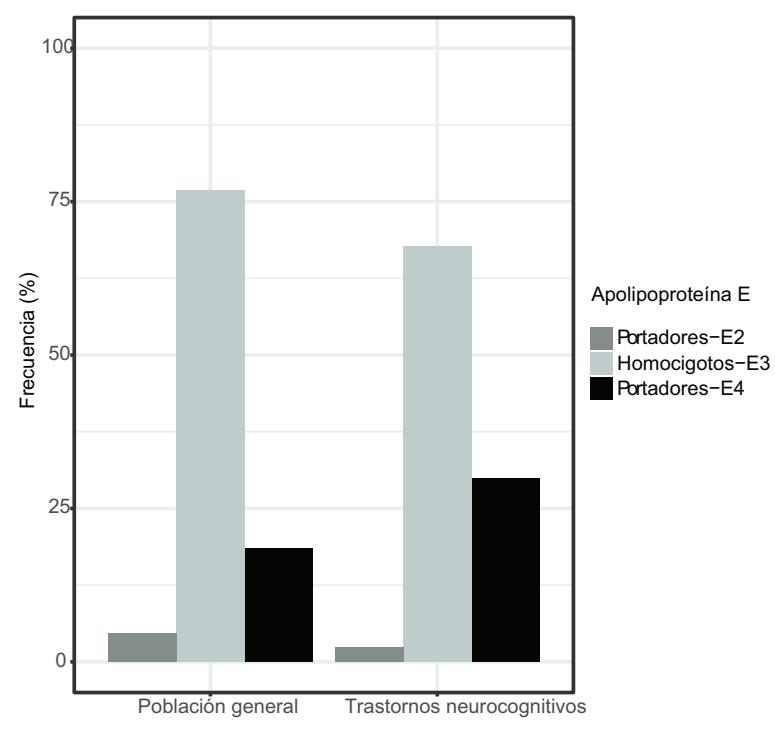

Figura 1. Distribución de APOE en población mexicana. El grupo de individuos con deterioro cognitivo presenta aumento en la frecuencia del alelo E4.

Hasta el momento se han evaluado genéticamente 297 sujetos mayores de 60 años y 70 familiares de primer grado de los sujetos con TNM. Los centros donde se ha implementado el programa son el Hospital Psiquiátrico "Fray Bernardino Álvarez", el Instituto de Neurología en Querétaro (Universidad Nacional Autónoma de México), el Hospital General de México y el Grupo Médico Carracci.

\section{Discusión}

La identificación previa a la manifestación de sintomatología de los individuos con alto riesgo genético para alguna enfermedad podría ayudar a disminuir las tasas de prevalencia. ${ }^{17}$ Por primera vez hemos implementado en México un programa de detección de individuos con alto riesgo genético de TNC con base en las isoformas del gen APOE.

Adicionalmente, con el objetivo de identificar un factor de riesgo de las isoformas de APOE con el TNC en población mexicana, se realizó un estudio de asociación del APOE-E4 con el TNC en esta población, ya que ha sido una de las relaciones más constantes y que más se ha replicado en diversas grupos humanos alrededor del mundo, ${ }^{18-20}$ no así en la mexicana. ${ }^{21}$

Tal como sucede en otras poblaciones, identificamos que en la mexicana el alelo APOE-E4 incrementó el riesgo para TNC, lo cual abre la puerta para plantear nuevas estrategias terapéuticas utilizando fármacos que emplean blancos moleculares relacionados con las isoformas de $A P O E^{22}$
Tabla 1. Comparación de las frecuencias de APOE en población mexicana

\begin{tabular}{|c|c|c|c|c|}
\hline & \multicolumn{2}{|c|}{ Población general $(n=1307)$} & \multicolumn{2}{|c|}{ TNC $(n=297)$} \\
\hline & $\mathrm{n}$ & $\%$ & n & $\%$ \\
\hline \multicolumn{5}{|c|}{ Genotipos de APOE } \\
\hline E2/E2 & 2 & 0.15 & 0 & 0.00 \\
\hline E2/E3 & 57 & 4.36 & 7 & 2.36 \\
\hline E2/E4 & 2 & 0.1 & 0 & 0.00 \\
\hline E3/E3 & 1005 & 76.89 & 201 & 67.68 \\
\hline $\mathrm{E} 3 / \mathrm{E} 4^{*}$ & 218 & 16.68 & 76 & 25.59 \\
\hline $\mathrm{E} 4 / \mathrm{E} 4^{*}$ & 23 & 1.76 & 13 & 4.38 \\
\hline \multicolumn{5}{|c|}{ Alelos de $A P O E$} \\
\hline E2 & & 2.41 & & 1.18 \\
\hline E3 & & 87.41 & & 81.64 \\
\hline E4 & & 10.10 & & 17.17 \\
\hline
\end{tabular}

En años recientes, como hemos planteado con anterioridad, se ha incrementado la idea de utilizar marcadores genéticos en el área clínica para ayudar en el diagnóstico e intervención terapéutica temprana. Una característica de las pruebas genéticas comparadas con otros métodos para la valoración de individuos con alto riesgo para TNC, como la resonancia magnética funcional, es su bajo costo. Sin embargo, se ha discutido si en países de bajos recursos y en desarrollo, como México, es factible implementarlas en la atención clínica y si pueden ser accesibles para toda la población. ${ }^{23}$

En este sentido, decidimos implementar este programa para tratar de incidir directamente en el área clínica y hacer más corta la brecha entre los datos genéticos y su implementación clínica, involucrando a los médicos, psicólogos y personal de apoyo que cuidan regularmente al paciente. Desafortunadamente, aun cuando el programa está funcionando en cuatro centros distintos del país, se requieren más esfuerzos para que pueda incidir en otros lugares y sea accesible económicamente a toda la población en riesgo, ya que a mayor demanda, costos más bajos.

\section{Conclusiones}

"Te APOyo" es el primer programa en México de detección de riesgo genético que confiere ser portador del alelo APOE-E4 para desarrollar trastorno neurocognitivo. En el análisis de asociación genética con la población general se replicaron análisis previos en los que el alelo APOE-E4 se estimó como factor de riesgo para TNC en población portadora del alelo.

Se espera que al conocerse portadores de alelo de riesgo, los participantes del programa cambien su estilo de vida en cuanto a alimentación, higiene del 
sueño y actividad física, entre otros aspectos, como se ha documentado en otros países, y que incluso se muestren optimistas por los resultados de la prueba; ${ }^{24}$ sin embargo, será necesario realizar seguimiento en nuestra población para saber su comportamiento.

De cualquier forma, se requiere mayor investigación básica para descubrir otras variantes genéticas que pudieran implementarse en el área clínica como marcadores de riesgo para TNC, adicionalmente a APOE-E4.

\section{Agradecimientos}

A Alejandro Velázquez y Erick Alejandro Izquierdo García, por su ayuda y entusiasmo durante la realización de este proyecto.

\section{Bibliografía}

1. Llibre-Rodríguez JJ, Ferri CP, Acosta D, Guerra M, Huang Y, Jacob KS, et. al. Prevalence of dementia in Latin America, India, and China: a population-based cross-sectional survey. Lancet. 2008;372(9637): 464-474.

2. Prince M, Bryce R, Albanese E, Wimo A, Ribeiro W, Ferri CP. The global prevalence of dementia: a systematic review and metaanalysis. Alzheimers Dement. 2013;9(1):63-75

3. Mejía-Arango S, Gutiérrez LM. Prevalence and incidence rates of dementia and cognitive impairment no dementia in the Mexican population: data from the Mexican Health and Aging Study. J Aging Health. 2011; 23(7):1050-1074

4. Stefanacci RG. The costs of Alzheimer's disease and the value of effective therapies. Am J Manag Care. 2011;17(Suppl13):S356-S362.

5. The Lancet Neurology. Pointing the way to primary prevention of dementia. Lancet Neurol. 2017;16(9):677.

6. Daviglus ML, Bell CC, Berrettini W, Bowen PE, Connolly ES, Cox NJ et al. NIH state-of-the-science conference statement: preventing Alzheimer's disease and cognitive decline. NIH Consens State Sci Statements. 2010;27(4):1-30.

7. Barnett JH, Lewis L, Blackwell AD, Taylor M. Early intervention in Alzheimer's disease: a health economic study of the effects of diagnostic timing. BMC Neurol. 2014;14:101.
8. Panegyres PK, Berry R, Burchell J. Early Dementia Screening. Diagnostics. 2016;6(1):6.

9. Hampel H, Broich K, Hoessler Y, Pantel J. Biological markers for early detection and pharmacological treatment of Alzheimer's disease. Dialogues Clin Neurosci. 2009;11(2):141-157.

10. Elias-Sonnenschein LS, Viechtbauer W, Ramakers IH, Verhey FR, Visser PJ. Predictive value of APOE- $\varepsilon 4$ allele for progression from $\mathrm{MCl}$ to AD-type dementia: a meta-analysis. J Neurol Neurosurg Psychiatry. 2011;82(10):1149-1156

11. Bertram L, McQueen MB, Mullin K, Blacker D, Tanzi RE. Systematic meta-analyses of Alzheimer disease genetic association studies: the AlzGene database. Nat Genet. 2007;39(1):17-23.

12. Qian J, Wolters FJ, Beiser A, Haan M, Ikram MA, Karlawish J, et al. APOE-related risk of mild cognitive impairment and dementia for prevention trials: an analysis of four cohorts. PLoS Med. 2017;14(3):e1002254.

13. Gutiérrez-Robledo LM, Arrieta-Cruz I. Dementia in Mexico: the need for a National Alzheimer's Plan. Gac Med Mex. 2015;151(5):667-673.

14. Diagnostic and statistical manual of mental disorders. Quinta edición. EE.UU.: American Psychiatric Association; 2013.

15. Folstein MF, Folstein SE, McHugh PR. "Mini-mental state". A practical method for grading the cognitive state of patients for the clinician. J Psychiatr Res. 1975;12(3):189-198.

16. Contreras-Cubas C, Sánchez-Hernández B, García-Ortiz H, Martínez-Hernández A, Barajas-Olmos F, Cid M, et. al. Heterogenous distribution of MTHFR gene variants among mestizos and diverse Amerindian groups from Mexico. PLoS One. 2016;11(9):e0163248.

17. Andermann A, Blancquaert I. Genetic screening: a primer for primary care. Can Fam Physician. 2010;56(4):333-339.

18. Ihle A, Bunce D, Kliegel M. APOE $\varepsilon 4$ and cognitive function in early life: a meta-analysis. Neuropsychology. 2012;26(3):267-277.

19. Agarwal R, Tripathi CB. Association of apolipoprotein $E$ genetic variation in Alzheimer's disease in Indian population: a meta-analysis. Am J Alzheimers Dis Other Demen. 2014;29(7):575-582.

20. Chen K, Sun YM, Zhou Y, Zhao Q, Ding D, Guo Q. Associations between APOE polymorphisms and seven diseases with cognitive impairment including Alzheimer's disease, frontotemporal dementia, and dementia with Lewy bodies in southeast China. Psychiatr Genet. 2016; 26(3):124-131.

21. Campos M, Edland SD, Peavy GM. An exploratory study of APOE-ع4 genotype and risk of Alzheimer's disease in Mexican Hispanics. J Am Geriatr Soc. 2013;61(6):1038-1040.

22. Yamazaki Y, Painter MM, Bu G, Kanekiyo T. Apolipoprotein E as a therapeutic target in Alzheimer's disease: a review of basic research and clinical evidence. CNS Drugs. 2016;30(9):773-789.

23. Maltese PE, Poplavskaia E, Malyutkina I, Sirocco F, Bonizzato A, Capodicasa N, et. al. Genetic tests for low- and middle-income countries: a literature review. Genet Mol Res. 2017;16(1).

24. Ashida S, Koehly LM, Roberts JS, Chen CA, Hiraki S, Green RC. Disclosing the disclosure: factors associated with communicating the results of genetic susceptibility testing for Alzheimer's disease. J Health Commun. 2009;76814(8):768-784. 


\section{Anexo 1. Formato de entrega de resultados.}

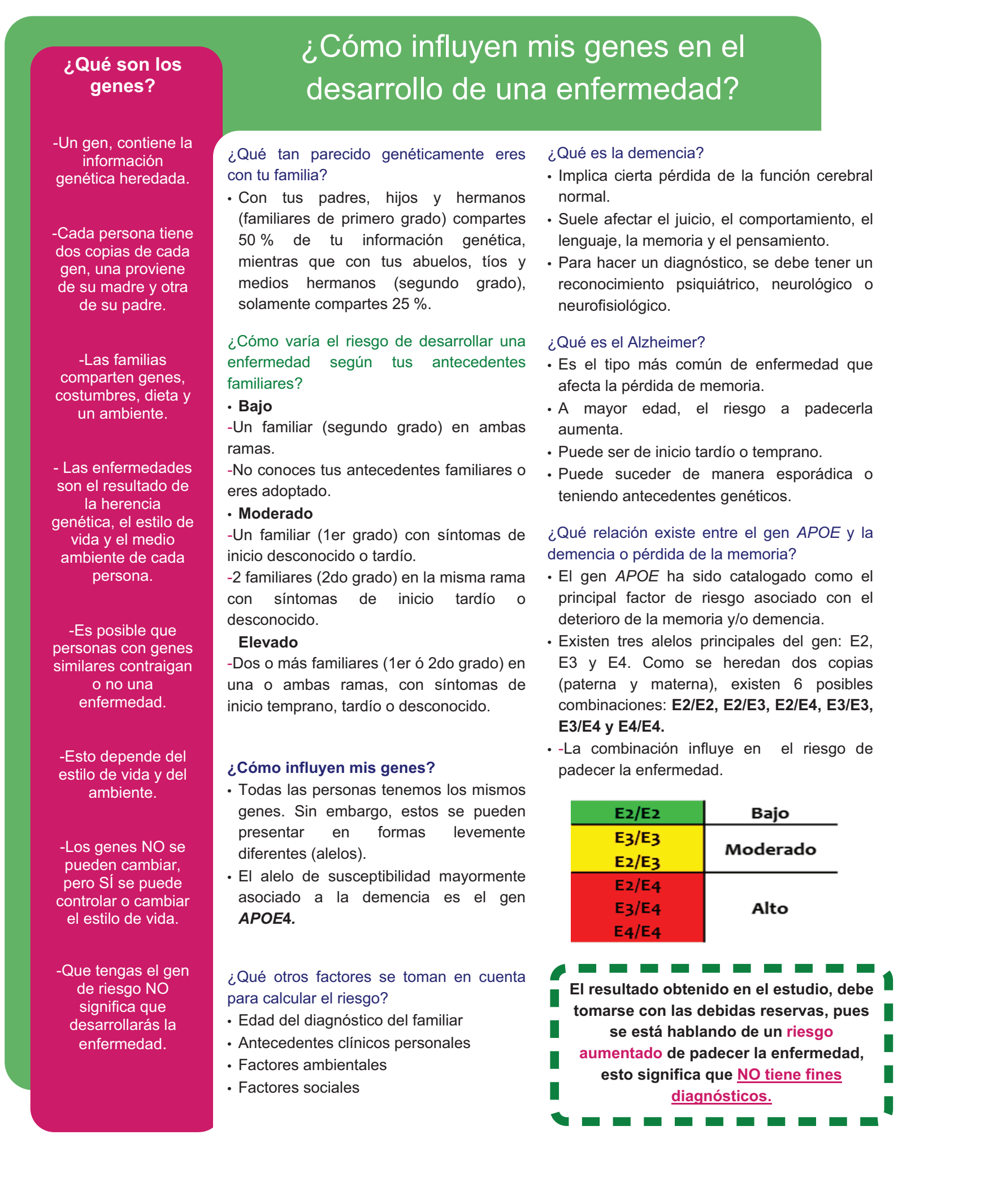

